\title{
A Study of Fatigue in Metals by Means of X-Ray Strain Measurement
}

\author{
John A. Bennett
}

\begin{abstract}
X-ray determinations of lattice strain were made on steel specimens under a series of static bending moments. These series of determinations were made on the specimens as normalized and after various amounts of fatigue stressing. The slope of the line relating the bending moment to the lattice strain was found to decrease with increasing numbers of cycles of alternating stress. However, the decrease was of approximately the same magnitude for specimens stressed above and below the fatigue limit, showing that the change was not associated with fatigue damage.
\end{abstract}

\section{Introduction}

In 1941 Glocker and coworkers ${ }^{1}$ in Germany, published a report on the study of fatigue damage by means of X-ray stress measurement. He claimed that as fatigue damage increased there was a change in the value of the surface stress measured with a given static load on the specimen. This claim, if verified, would have required a fundamental change in the prevalent ideas regarding the mechanism of fatigue, according to which fatigue damage occurs in a very small volume of the metal and the rest of the material remains unchanged. This point of view would have to be abandoned, if it were possible to detect fatigue damage by means of X-ray diffraction, as the diffraction occurs from a relatively large volume close to the surface of the specimen.

Because certain features of Glocker's work made the results open to question, a project was initiated with a view to reproducing his experiments. It was immediately apparent at that time that in order to obtain an unambiguous result the sensitivity of stress measurement would have to be improved over that which could be realized with the methods available. The techniques and instruments that have been developed in achieving this objective have been described previously. ${ }^{23}$ The improvement in sensitivity and accuracy was made possible by increasing the number of measurements made on each diffraction ring and by the use of instrumental rather than visual methods for making the measurements.

\section{Materials and Test Methods}

The specimens used in this work were made from SAE 4130 steel having the following composition (in percent): C, $0.33 ; \mathrm{Mn}, 0.53 ; \mathrm{P}, 0.013 ; \mathrm{S}, 0.019$; Si, 0.24; Cr, 0.91; Mo, 0.21. This steel was from the heat used for tests reported previously, ${ }^{4}$ being identified in that report as material M357. The

IJR. Glocker, W. Lutz, and O. Schaaber, Evidence on the state of fatigue of metals obtained from an examination of the surface stresses by means of X-rays,

Z. Ver. deut. Ing. 85, 793 (Oct. 1941).
2 J. A. Bennett and H. C. Vacher, Calibration of X-ray measurement of strain, J. Research NBS 40, 285 (1948) RP1874.

$3 \mathrm{~J}$. A. Bennett, Instrument for measurement of X-ray diffraction patterns, Rev. Sci. Instr. 20, 908 (1949).

4 J. A. Bennett, A study of the damaging effect of fatigue stressing on X4130 steel, Proc. Am. Soc. Testing Mat. 46, 693 (1946); J. Research NBS 37, 123 (1946) RP1733. grain size was small, corresponding to No. 10 on the ASTM scale, which made the steel particularly suited to X-ray diffraction work.

The specimens were of the commonly used R. R. Moore type having a minimum diameter of 0.25 in. and were heat-treated after machining and polishing in order to leave the surface free from plastic deformation. The polishing was done manually with Aloxite paper, the direction of polishing being parallel to the length of the specimen. After they were polished, the specimens were heated in vacuum to $1,650^{\circ} \mathrm{F}$ and held $1 \mathrm{hr}$. They were then cooled by removing the furnace from around the vacuum chamber, resulting in a cooling rate nearly as rapid as air cooling. This treatment will be referred to as normalizing in the remainder of this report. Finally, the reduced section was etched 2 min in 1-percent nital, this having been found necessary to obtain a sharp diffraction pattern.

The procedure used in all the tests reported here was to measure the lattice strain under a series of applied static loads. These series of determinations were made on the specimen in the normalized condition and after various amounts of fatigue stressing. The strain determinations were based on the changes in the diffraction angle between patterns taken with the X-ray beam incident at two different angles.

The jig for applying the static load to the fatigue specimen is shown in figure 1. By rotating the loading screw, either a tensile or compressive stress could be applied to the front surface of the specimen. The load was applied through two calibrated springs (only one of which can be seen in the figure) and a pair of yokes that distributed the load symmetrically to the two pins in the specimen adapters. The deflections of the springs were measured with a dial indicator having a long projection, which was inserted through a hole in the shank of the spring.

The springs were calibrated by dead-weight loading and the bending moment on the specimen was calculated from this calibration and the measured distance between the pairs of pins in the specimen adapters. Near the end of the investigation reported here, this calibration was checked by means of a specimen having a rectangular cross section between the tapers. Wire strain gages were mounted on this specimen and the bending moment applied to the specimen in the jig was measured directly. This 


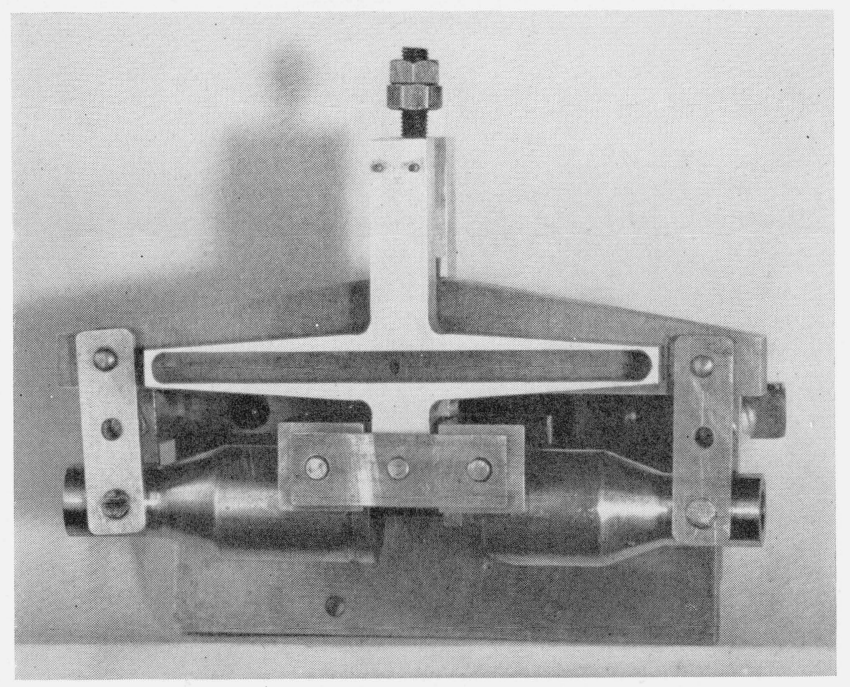

Figure 1. Loading jig for applying a static load to the fatigue specimen.

bending moment was in good agreement with that calculated from the spring deflection.

The loading jig was mounted on the assembly described in the reference in footnote 2 , which provided means for setting the angle between the specimen and the X-ray beam and for traversing the specimen during the exposure. The film was not oscillated.

The technique for measuring the diffraction angle is described in detail in the references given in footnotes 2 and 3, so only a brief outline will be given here. The following symbols and definitions are used:

$R=$ radius of the $K \alpha_{1}$ diffraction ring

$S=$ specimen-to-film distance

$\theta=$ diffraction angle

$\tan 2 \theta=R / S$

$\beta=$ angle around the diffraction ring

$C=$ diameter of the calibrating ring

$\epsilon=$ strain in a given direction

$\epsilon_{x}=$ strain in a direction parallel to the stress

Strain ratio $=\epsilon / \epsilon_{x}$.

The quantity $\tan 2 \theta$ was determined by measuring $R$ and $S$. The measurement of $S$ was accomplished by means of a micrometer mounted on a separate camera track, the whole specimen mount assembly being placed on this track before and after making an exposure. The radius, $R$, was determined by measurements on the diffraction patterns; a calibrating ring was exposed on the film immediately after the diffraction ring, and the distance between the two measured. This measurement, corrected for film shrinkage, was subtracted from the known inside diameter of the calibrating ring to obtain the radius of the $K \alpha_{1}$ ring.

A sketch of the instrument used for making these measurements is shown in figure 2 and is fully described in the reference cited in footnote 3 . It is essentially a densitometer having a dial indicator to measure the movement of the film. The slit in the optical system is curved so that the beam of light

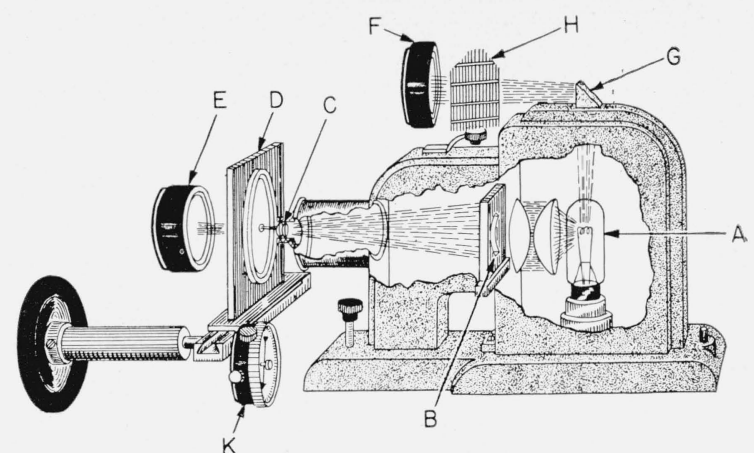

FiguRE 2. Instrument for measurement of diffraction patterns. A, Lamp; B, slit; C, 24-mm microscope objective; D, film holder; E, meas ur. ing photocell; $\mathrm{F}$, compensating photocell; $\mathrm{G}$, prism; H, adjusting screen; $\mathrm{K}$, dial indicator

that scans the diffraction ring is an are having the same radius of curvature as the ring.

For each stress determination two patterns were exposed, one with the X-ray beam incident normal to the specimen and the other with an incident angle of $45^{\circ}$. In the latter case the incident beam, the axis of the specimen and the normal to its surface are in the same plane. Twelve measurements of ring radius were made on each pattern; for the $90^{\circ}$ incidence patterns the ring radius would be expected to be nearly constant around the ring, so the 12 readings were averaged. For the oblique incidence patterns, however, the radius would theoretically vary around the ring except for conditions of zero stress. However, by plotting the measurements on these patterns against strain ratio, the points would be expected to fall on a straight line so the intercept at $\beta=0$ as determined by least squares can be taken as the value representative of the 12 readings. This method is applicable only in the case of uniaxial stress.

Since the publication of the reference cited in footnote 2 , it has been found necessary to make a correction for misalinement between the X-ray beam and the center of the calibrating ring. The magnitude of this correction is determined from the deviations of individual readings from the average for patterns taken with the beam normally incident. Figure 3 shows a plot of these deviations against the angular position on the diffraction ring. The value plotted at each angular position is the average deviation observed on six patterns. The assumption is made that the diffraction ring is a circle and that the observed deviations are due to a displacement between the center of this ring and the center of the calibrating ring. The correction would then be a sine curve, and such a curve has been drawn on figure 3, the phase and amplitude having been adjusted visually to give the best apparent fit to the observed points.

The correction mentioned above was made rather than trying to eliminate the misalinement because of the small size of the error. It will be noted that the data shown in figure 3 , which are typical, indicate a displacement (between the center of the diffraction 


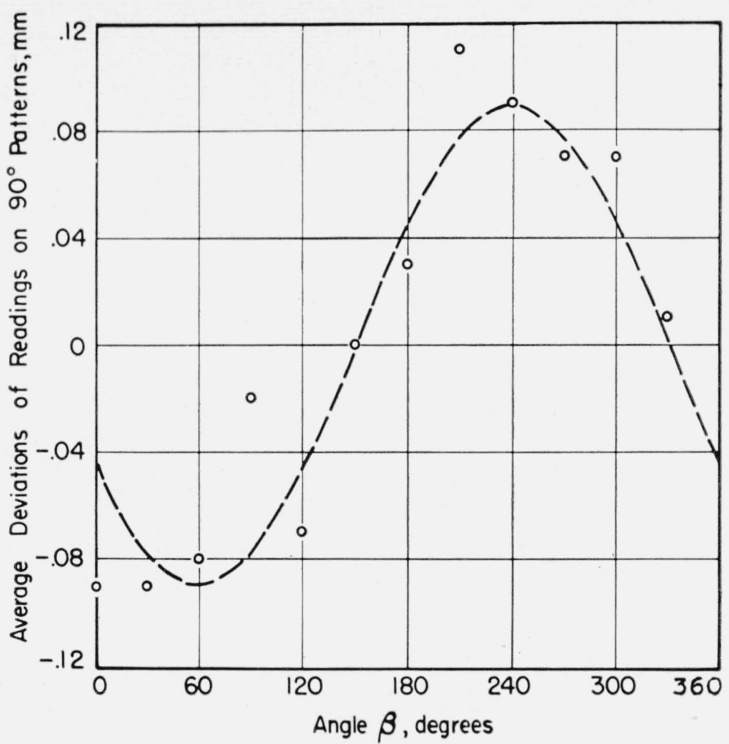

FIgURE 3. Deviations of individual readings from average for patterns taken at normal incidence.

ring and the center of the calibrating ring) of less than $0.1 \mathrm{~mm}$. The collimator for the X-ray beam has pinholes with a diameter of $1 \mathrm{~mm}$, so it appeared probable that one of the causes of misalinement was uneven illumination from the target, which would cause the center of gravity of the beam to be slightly displaced from the center of the pinholes. This was corroborated by the fact that the deviations changed when it was necessary to change the X-ray tube. As this source of error was inherent in the X-ray apparatus it was not thought worth while to attempt to reduce the other factors that contributed to the total misalinement.

This correction, of course, made no difference in the average values of the radius obtained from measurements on the patterns taken at normal incidence. However, there was a significant change in the intercept obtained from the measurements on the oblique incidence patterns (when the corrections obtained from the normal incidence patterns were applied). Figure 4 shows the measurements, corrected for misalinement, from a typical pattern taken at $45^{\circ}$ incidence. The points are plotted on a uniform strain ratio scale as shown at the top of the figure so that they would be expected to lie on a straight line, and it can be seen that this expectation is approximately fulfilled.

\section{Results}

The above procedure, then, provides a single value of $\tan 2 \theta$ representative of each pattern. Figure 5 shows these values plotted against the bending moment applied to the specimen for a typical series of determinations. A positive value was assigned to the bending moment when the irradiated surface of the specimen was in tension, and vice versa. The order of applying the loads was as follows: 0,15 ,

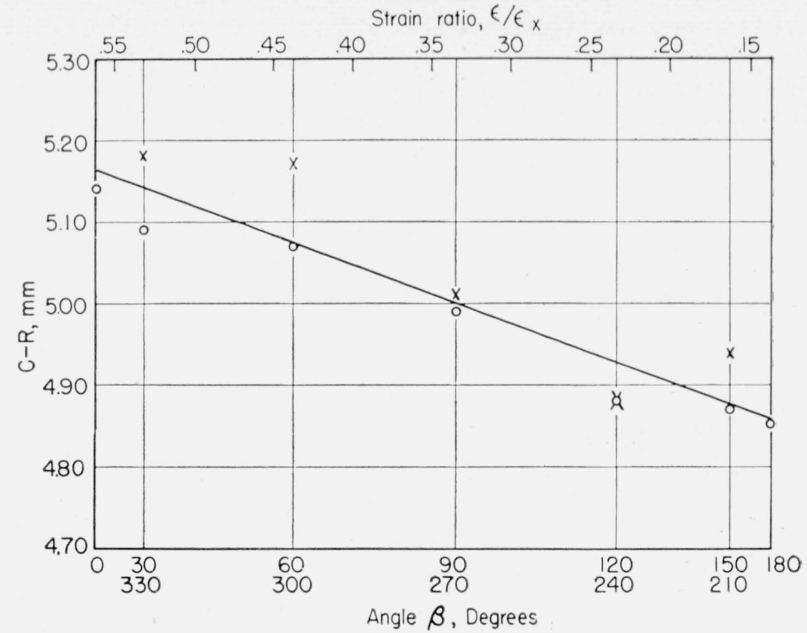

FiguRE 4. Typical plot of the measurements on one diffraction. pattern.

, Values from $0^{\circ}$ to $180^{\circ} ; \mathrm{x}$, values from $210^{\circ}$ to $330^{\circ}$.

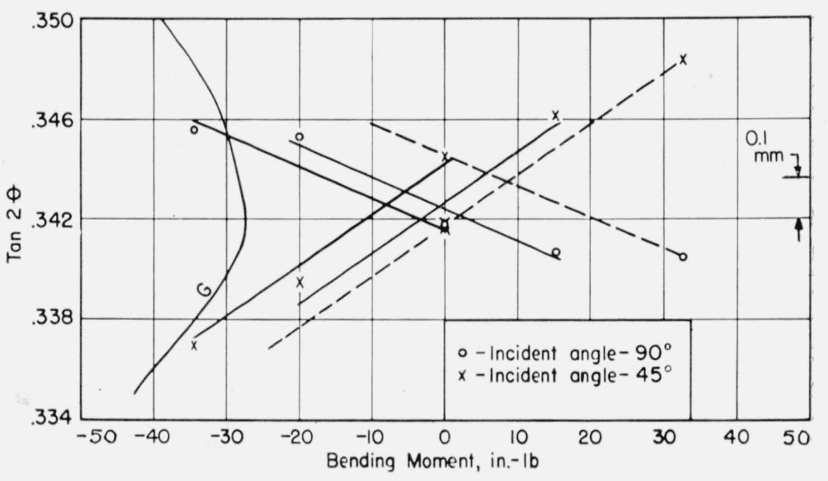

Figure 5. Effect of bending moment on diffraction angle.

The scale on the right shows equivalent distance on the film for a specimen-tofilm distance of $60 \mathrm{~mm}$. The curve $\mathrm{G}$ shows the galvanometer dentections obtained in scanning a typical $K \alpha_{1}$ line. The ordinate scale for this curve is the one at the right; the absissa seale is arbitrary except that the left edge
corresponds to the minimum density between the $K \alpha_{1}$ and $K \alpha_{2}$ lines.

$-20,33,-35,0$ in.-lb. In general, a similar schedule was followed in all the series of strain determinations reported here; the maximum load in either tension or compression was of such magnitude as to cause a nominal stress of approximately $30,000 \mathrm{lb} / \mathrm{in} .{ }^{2}$ in the outer fibers of the specimen, the intermediate loads being about half as large.

In order to give an idea of the relationship between the ordinates on figure 5 and actual dimensions on the diffraction patterns, the change in $\tan 2 \theta$ corresponding to $0.1-\mathrm{mm}$ displacement on the pattern is indicated at the right of the figure. On the left side of the graph is a curve $(G)$ representing the galvanometer deflections in the pattern-measuring instrunoment which were observed in traversing a typical $K \alpha_{1}$ line. The zero point for this curve was taken as the minimum density observed between the $K \alpha_{1}$ and $K \alpha_{2}$ lines, and this minimum is represented by the left edge of the graph. That is, the total height of the graph represents a change of $\tan 2 \theta$ considerably less than the width of the diffraction line. 
Previous work (footnote 2) had shown that the most convenient measure of the lattice strain was the difference in the values of $\tan 2 \theta$ between the patterns taken with the beam incident at $45^{\circ}$ and those taken at normal incidence. This difference $\left(\tan 2 \theta_{B}-\tan 2 \theta_{A}\right)$ is shown in figure 6 plotted against the bending moment applied to the specimen. From a study of a number of graphs of this type, it was possible to determine which changes from linearity in the experimental points were due to plastic deformation in the specimen and which were simply scatter. The numbers adjacent to the points represent the order in which the respective loads were applied. The location of the points and the reasons for drawing the lines in the particular manner shown can be explained as follows: for points 2 and 3 the applied loads caused only elastic deflection of the specimen, so the lattice strain was proportional to the bending moment in this range. However, when the moment was increased above a certain point, plastic deformation began to take place on one or both surfaces of the specimen, and there was little or no further increase in lattice strain, therefore point 4 is offset to the right of the line joining points 1,2 , and 3 . When the high positive moment was reversed, it would be expected that the locus of the plotted values would follow the elastic relationship shown by the dotted line. On reaching a negative lattice strain of about the same magnitude as that at which plastic deformation occurred in the positive direction, the increase in elastic strain again practically stopped so that point 5 is offset to the opposite side of the original elastic line. On release of this moment, the values again followed an elastic line parallel to the original one, the final point 6 , representing a residual tension in the surface of the specimen under study.

The above appears to be a logical interpretation of the observed data, and the practice was adopted

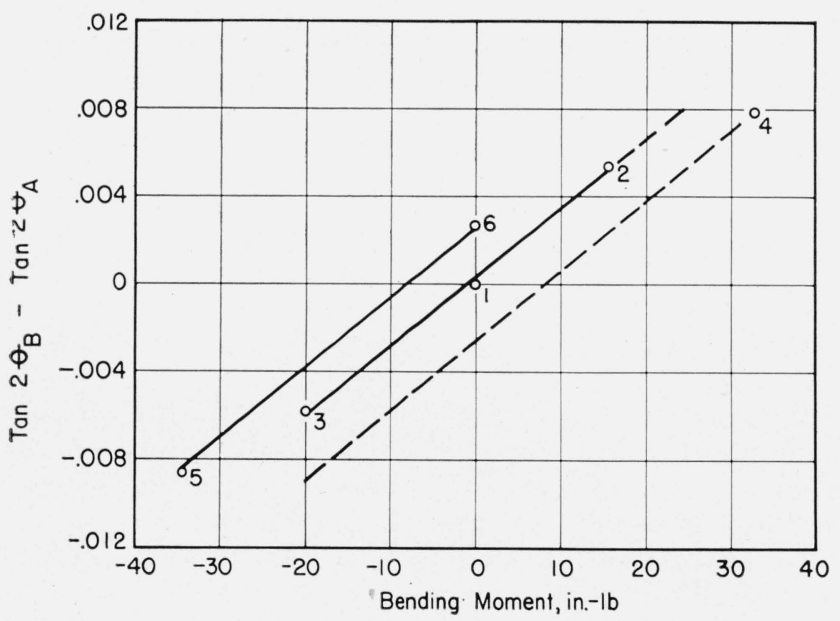

FIGURE 6. Difference between the $90^{\circ}$ and $45^{\circ}$ points shown in figure 5.

The subscripts $A$ and $B$ indicate values of $\tan 2 \theta$ obtained with $90^{\circ}$ and $45^{\circ}$ incidence, respectively. of determining the slope of the elastic line for a given condition by averaging the slope between points 2 and 3 with that between 5 and 6 . On returning to figure 5 and drawing lines determined in this way for both sets of data, it was found that the deviation of the points from the lines was reasonably small. All other methods of analyzing the data that were investigated resulted in deviations that were larger than would be expected in view of the precision of the data.

This average slope appears to be the characteristic of the data obtained in this investigation that most nearly corresponds to the composite slope that Glocker measured. Accordingly, in order to test the correctness of his hypothesis, the value of this slope was plotted against the number of stress cycles to which the specimen had been subjected when the series of strain determinations were made. Figures 7 and 8 show these graphs for specimens stressed above and below the fatigue limit, respectively, the ordinate being expressed in terms of the percentage of the slope for the normalized material. The values of the slopes for these two specimens are given in table 1.

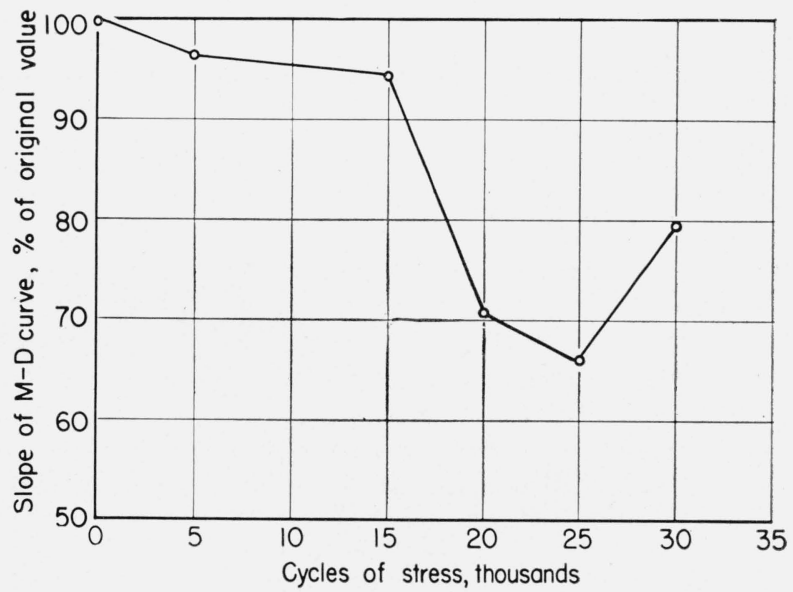

FIGURE 7. Change in the relation between bending moment and lattice strain for a specimen stressed in fatigue at an amplitude of $54,000 \mathrm{lb} / \mathrm{in}^{2}$

Fatigue crack formed between 35 and 40 thousand cycles.

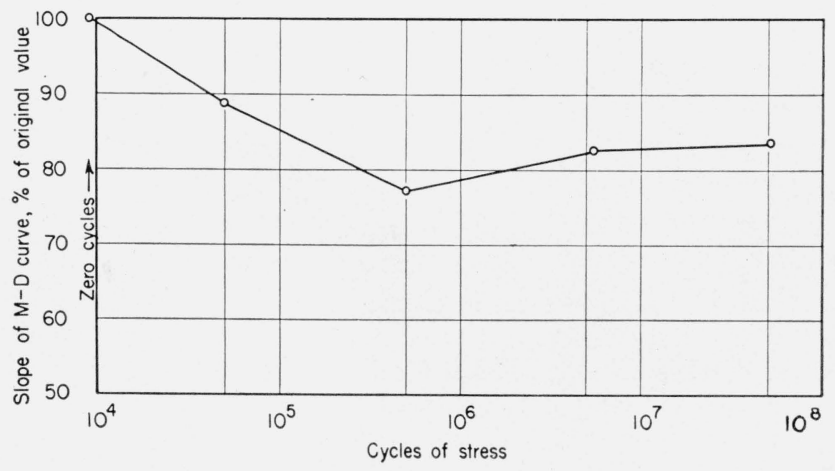

FIgURE 8. Change in the relation between bending moment and lattice strain for a specimen stressed in fatigue at an amplitude of $35,000 \mathrm{lb} / \mathrm{in}^{2}$ 
TABLE 1. Effect of fatigue stressing on the relation between applied bending moment and lattice strain

\begin{tabular}{|c|c|}
\hline Condition & $\begin{array}{l}\text { Slope of } \\
\text { M-D } \\
\text { curve a }\end{array}$ \\
\hline \multicolumn{2}{|c|}{ b A. Fatigue stress amplitude $54,000 \mathrm{lb} / \mathrm{in}^{2}{ }^{2}$} \\
\hline $\begin{array}{l}\text { As normalized } \\
\text { After } 5,000 \text { cycles } \\
\text { After } 15,000 \text { cycles. } \\
\text { After } 20,000 \text { cycles } \\
\text { After } 25,000 \text { cycles. } \\
\text { After } 30,000 \text { cycles. }\end{array}$ & $\begin{array}{l}(\text { in. }-l b)^{-1} \\
3.02 \times 10^{-4} \\
2.91 \\
2.85 \\
2.13 \\
1.99 \\
2.40\end{array}$ \\
\hline \multicolumn{2}{|c|}{ B. Fatigue stress amplitude $35,000 \mathrm{lb} / \mathrm{in}^{2}$} \\
\hline $\begin{array}{l}\text { As normalized } \\
\text { After } 5 \times 10^{4} \text { cycles } \\
\text { After } 5 \times 10^{5} \text { cycles } \\
\text { After } 5.5 \times 10^{6} \text { cycles } \\
\text { After } 5.1 \times 10^{7} \text { cycles }\end{array}$ & $\begin{array}{l}\text { 3. } 90 \times 10^{-4} \\
\text { 3. } 46 \\
3.01 \\
3.22 \\
3.25\end{array}$ \\
\hline \multicolumn{2}{|c|}{ (Fatigue stress amplitude $54,000 \mathrm{lb} / \mathrm{in} .{ }^{2}$} \\
\hline After 25,000 cycles.... & 3.06 \\
\hline
\end{tabular}

a The $\mathrm{M}$-D curve represents the relationship between the applied bending moment and the lattice strain (as measured by the difference in $\tan 2 \theta$ for normal and oblique patterns).

b A fatigue crack formed in this specimen between 35,000 and 40,000 cycles

A subsequent test, not shown on figure 8, was performed on the specimen that had run 51 million cycles at $35,000 \mathrm{lb} / \mathrm{in}^{2}$ The stress was raised to $54,000 \mathrm{lb} /$ in. $^{2}$ and the specimen run 25,000 cycles before making a series of strain determinations. The slope obtained from this series was 78 percent of the original value. This is above the lowest value obtained during stressing below the fatigue limit, and so close to the value after $51 \times 10^{6}$ cycles that the change can hardly be considered significant. The fatigue crack formed in this specimen between 35,000 and 40,000 cycles.

\section{Discussion}

It is believed that figures 7 and 8 provide the answer to the original problem on which this work was based; it can be seen that there is a decrease in slope for both conditions, and the magnitude of the decrease is not much greater for the specimen stressed above the fatigue limit than for that stressed below. The change, therefore, cannot be said to be due to fatigue damage. This is further verified by the subsequent test on the specimen stressed below the fatigue limit. The conditions that caused the greatest decrease for the specimen stressed originally above the fatigue limit caused a decrease of only 5 percent in the slope for the specimen that had had prior fatigue stressing.

While the primary problem for which the project was initiated has been solved, this work has raised several new questions and has pointed the way to other possibly fruitful lines of research. For example, it is surprising that a specimen that has been subjected to millions of cycles of stress with a maximum value of $35,000 \mathrm{lb} / \mathrm{in}^{2}$ (the data of figs. 5 and 6 represent this condition) can be deformed plastically by a static stress less than $30,000 \mathrm{lb} / \mathrm{in} .{ }^{2}$ The further investigation of this anomaly would undoubtedly provide valuable information on the mechanisms of plastic deformation and fatigue.

\section{Summary}

1. Determinations were made of the relationship between the bending moment applied to the specimen and the lattice strain in the most highly stressed fibers as obtained by X-ray measurements. The slope of the curve representing these measurements usually decreased with increasing amounts of fatigue stressing applied before the determinations were made.

2. The greatest decrease in slope was of the same order of magnitude for specimens stressed above and below the fatigue limit. A specimen that had run $51 \times 10^{6}$ cycles at a stress below the fatigue limit was subsequently stressed for about 70 percent of its life at a stress amplitude above the fatigue limit. Almost no change in the slope was found after this damaging treatment.

3. These determinations, therefore, do not appear to give any correlation with fatigue damage.

4. The data indicate that even after millions of cycles of fatigue stressing plastic deformation occurred under static stress less than the prior fatigue stress.

The author is indebted to Lura F. Roehl, who made the diffraction patterns and carried out the extensive measurements and calculations involved in this study. He also wishes to thank H. C. Vacher for many helpful suggestions in regard to the X-ray techniques used.

Washington, September 15, 1950. 